\title{
Robot-aided neurorehabilitation of the upper extremities
}

\section{Review Article}

\section{Author(s):}

Riener, Robert; Nef, Tobias; Colombo, Gery

Publication date:

2005-02

Permanent link:

https://doi.org/10.3929/ethz-b-000411918

Rights / license:

In Copyright - Non-Commercial Use Permitted

Originally published in:

Medical \& Biological Engineering \& Computing 43(1), https://doi.org/10.1007/BF02345116 


\title{
Robot-aided neurorehabilitation of the upper extremities
}

\author{
$\begin{array}{lll}\text { R. Riener } & \text { T. } \text { Nef }^{1,2} & \text { G. Colombo } \\ & \text { 2,3 }\end{array}$ \\ ${ }^{1}$ Rehabilitation Engineering Group, Automatic Control Laboratory, Swiss Federal Institute of \\ Technology $(\mathrm{ETH})$, Zurich, Switzerland \\ ${ }^{2}$ Spinal Cord Injury Center, University Hospital Balgrist, University Zurich, Switzerland \\ ${ }^{3}$ Hocoma AG, Volketswil, Switzerland
}

\begin{abstract}
Task-oriented repetitive movements can improve muscle strength and movement co-ordination in patients with impairments due to neurological lesions. The application of robotics and automation technology can serve to assist, enhance, evaluate and document the rehabilitation of movements. The paper provides an overview of existing devices that can support movement therapy of the upper extremities in subjects with neurological pathologies. The devices are critically compared with respect to technical function, clinical applicability, and, if they exist, clinical outcomes.
\end{abstract}

Keywords-Neurorehabilitation, Movement therapy, Robotics, Paralysis, Stroke, Upper extremities

Med. Biol. Eng. Comput., 2005, 43, 2-10

\section{Introduction}

\subsection{Clinical background of arm therapy}

ARM THERAPY is used in neurorehabilitation for patients with paralysed upper extremities due to lesions of the central or peripheral nervous system, e.g. after stroke or spinal cord injury. The goal of the therapy is to recover motor function, improve movement co-ordination, learn new motion strategies ('trick movements') and/or prevent secondary complications, such as muscle atrophy, osteoporosis and spasticity.

Several studies have proved that arm therapy has positive effects on the rehabilitation progress of stroke patients (see PlATZ (2003) for a review). Many researchers have compared the efficiency of different therapeutic approaches (BASMAJIAN et al., 1987; DICKSTEIN et al., 1986; LORD and HALL, 1986; WAGENAAR et al., 1990). In general, there is a positive effect on the patient's progress in rehabilitation with each therapeutic approach. However, no significant differences in the levels of efficiency can be found between the different approaches. LANGHAMMER and STANGHELLE (2000) presented one exception, where a group of stroke patients treated with a taskoriented 'motor-relearning programme' showed improved motor functions compared with another group of patients undergoing a Bobath therapy.

Besides these classical approaches, innovative therapies have been developed in recent years demonstrating distinct efficiency for specific patient subgroups. Such approaches include constrained-induced movement therapy for patients with partial functional deficits (TAUB et al., 1993), as well as repetitive training techniques (FEYs et al., 1998), electromyographical

Correspondence should be addressed to Professor Robert Riener; email: riener@control.ee.ethz.ch

Paper received 16 June and in final form 22 July 2004

MBEC online number: 20053947

(C) IFMBE: 2005 biofeedback (SCHLEENBAKER and MAINOUS, 1993) and functional electrical stimulation (e.g. SONDE et al., 1998) for patients with severe arm paresis.

Several groups observed that longer training sessions per week and longer total training periods have a positive effect on the motor function of the arm (SUNDERLAND, 1992; KWAKKEL et al., 1999; 2002). In a meta-analysis comprising nine controlled studies with 1051 stroke patients, KWAKKEL et al. (1997) showed that increased training intensity yields moderate positive effects on neuromuscular function and ADL. This study did not distinguish between upper and lower extremities. The finding that the rehabilitation progress depends on training intensity supports the application of robot-aided arm therapy.

\subsection{Classification of rehabilitation robots}

Task-oriented repetitive movements can improve muscle strength and movement co-ordination in patients with neurological impairments. Robots can support movement therapy of the lower and upper extremities. In the past, several robotbased approaches were presented to support the rehabilitation of neurologically impaired subjects.

Two groups of robotic aids can be distinguished. First, there are therapeutic systems that are mainly used in a clinical environment, thus being shared by several patients. The second group are home-use systems that assist a single patient in activities of daily living. They range from wheelchairs and mobile service robots to assistive manipulators that can be mounted onto wheelchairs or desks. Many of these systems are commercially available and were developed in the 1980s (KWEE et al., 1988; LEIFER, 1981; VAN DER LOOS et al., 1988).

This review focuses only on the therapeutic systems. They can be split into passive, active and interactive systems. In passive systems, no actuation is implemented to move patient limbs. Instead, limbs are passively stabilised, fixed or limited 
in the range of motion. Typical technical components are stiff frames, bearings and pulleys and ropes with counter-weights. Active systems are equipped with electromechanical, pneumatic, hydraulic and other drives to move patient limbs actively. Either the devices are open-loop controlled, or simple position-control strategies are implemented. Interactive systems are characterised not only by actuators but also by sophisticated impedance and other control strategies that allow reaction to the patient efforts.

Impedance controllers are well-established in the field of robotics and human-system interaction. Hogan first introduced them about 20 years ago (HOGAN, 1985). The basic idea of the impedance control strategy applied to robot-aided therapies is to allow a variable deviation from a predefined leg trajectory rather than imposing a rigid gait pattern. The deviation depends on the patient's effort and behaviour. However, other control strategies are also possible to allow robot-patient interaction (RIENER and FUHR, 1998; JEZERNIK et al., 2003). Interactive systems require position and/or force sensors to measure the user-machine interaction and feed the controllers.

\subsection{Clinical requirements from a rehabilitation robot}

Clinical requirements can be divided into psychological, medical and ergonomic aspects. Psychological aspects have to be considered so that both therapist and patient are motivated. The therapist plans the rehabilitation process, explains the device function to the patient, adjusts the robot to the patient and performs the training with the aid of the robot. Despite the presence of a robot, the therapist remains the person in whom the patient has confidence. The therapist is the key person for a successful rehabilitation process, whereas the robot just supports the therapy defined by the therapist. Therefore the robot should remain rather 'invisible', so that the interaction between patient and therapist is not disturbed or destroyed. Consequently, the therapist should be involved right from the beginning of the robotic therapy. Furthermore, the robot should look 'human-friendly' and behave accordingly (ZINN et al., 2004), i.e. it should be safe, as small and lightweight as possible, 'friendly looking', quiet and compliant, just as the therapist's hand is during manual therapy. Neither therapist nor patient should be afraid of the robot.

In the design or application of rehabilitation robots, medical aspects must also be taken into account to ensure a successful training. It is crucial that the robot is adapted or adaptable to the human limb in terms of segment lengths, range of motion and the number of degrees-of-freedom (DOFs). A high number of DOFs allows a broad variety of movements, with many anatomical joint axes involved. However, this could make the device complex, inconvenient and expensive. It remains open how many DOFs are optimum. The question is whether the therapeutic outcome can be maximised, if the robot acts on the entire extremity rather than on single joints only. It may take until the end of a training session or clinical study performed with a specific device to answer this question. However, there is evidence that a therapy that focuses on activities of daily living (ADLs) not only increases patient motivation but also yields an improved therapeutic outcome, compared with single joint movements (LANGHAMMER and STANGHELLE, 2000). This kind of therapy is also called a 'motor relearning programme'

Furthermore, when new robotic devices are being designed, clinical standards must be considered, to retain compatibility with traditional therapies. Therefore the robot-aided therapy should provide the therapist with well-known scores for the evaluation of patient status and rehabilitation progress (e.g. Asworth scale, Fugl-Meyer score). As the robot replaces the therapist's hand, sophisticated sensor systems should be integrated to measure the patient's muscular effort and movement. The measured data should be processed and presented to the therapist, so that she or he can assess the rehabilitation process.

There are several ergonomic or logistic challenges. The rehabilitation robot set-up must be rather flexible to cope with a large variety of different applications and situations. Patients of either gender and different body heights and weights must be able to use the device. Furthermore, it must be taken into account that the robot has to share space with additional equipment accompanying the patient. For example, during the rehabilitation phase, the robot must cope with different types of wheelchair and respiratory equipment. Additionally, it would be advantageous if bed-ridden patients could also use the system, as many patients are in a supine position before being transferred to a wheelchair. Last, but not least, it is fundamental to ensure that the robotic system is easy to use, because the technical background and the time of the therapist are usually limited. The modifications necessary to adjust the system to a patient must be as simple as possible.

\section{Technical overview of arm robots}

Table 1 gives an overview of existing robotic systems applicable for therapy of the upper extremities. The systems are ordered according to the degree of activity (passive, active, interactive systems) and the number of DOFs.

\subsection{Passive and active systems}

The Swedish Helparm* is a passive system based on counterweights that are connected to the patient's arms by ropes and pulleys to support the weight of the patient's arms during reaching tasks (Fig. 1). Left and right arm supports can be used independently of each other. The amount of support can be changed in discrete steps to adjust the device to the individual arm weight with varying amounts of assistance or resistance. The Swedish Helparm provides functional assistance and allows muscle training for patients with shoulder muscle impairment or paresis, cervical spine injuries, shoulder nerve injuries, hemiplegia, multiple sclerosis or certain forms of rheumatoid arthritis. It can be used to practise ADLs in the clinic and can assist the patient at home.

Baltimore Therapeutic Equipment (BTE) Co. produces several training devices that are based on a single-DOF drive and allow the patient to train different functional movements of the lower and upper extremities. Basic closed-loop control strategies give a system with adjustable viscous and elastic force characteristics. The BTE devices can be used for the training and evaluation of a wide variety of movements, including many different work and ADL tasks, as well as warming-up and stretching exercises (Fig. 2).

REINKENSMEYER et al. (1999a) developed a system called the assisted rehabilitation and measurement (ARM) guide. Compared with the other systems presented above, the ARM guide does not primarily support arm movements during therapy. Instead, it can be applied to the evaluation of arm impairments of subjects with chronic brain injury. The ARM guide can be classified as a passive system, as it does not involve any movement actuation. It has one passive, translational DOF that allows hand movements towards and away from the shoulder along a linear track. Another semi-active, rotational DOF corresponds to shoulder rotation in the vertical plane. This DOF is equipped with a brake that provides scalable resistance torques to hold the arm at a fixed elevation

*Kinsman Enterprises, Inc. 


\begin{tabular}{|c|c|c|c|c|c|}
\hline & Application & $\begin{array}{l}\text { Level of } \\
\text { interactivity }\end{array}$ & Active DOFs & $\begin{array}{l}\text { Reported number of } \\
\text { patients treated }\end{array}$ & References \\
\hline Swedish Helparm & training of ADL tasks & passive & 0 & unknown & - \\
\hline BTE devices & $\begin{array}{l}\text { training of different } \\
\text { ADL and work } \\
\text { tasks }\end{array}$ & active & 1 & unknown & - \\
\hline ARM-guide & $\begin{array}{l}\text { evaluation of chronic } \\
\text { brain-injured } \\
\text { patients }\end{array}$ & passive/semi-active & 1 & 5 & $\begin{array}{l}\text { REINKENSMEYER } \text { et } a l . \\
\quad(1999 a ; b)\end{array}$ \\
\hline $\begin{array}{l}\text { Hand-object-hand } \\
\text { rehabilitator }\end{array}$ & $\begin{array}{l}\text { treatment of } \\
\text { hemiparetic } \\
\text { patients }\end{array}$ & interactive & 1 & unknown & LUM et al. (1993) \\
\hline $\begin{array}{l}\text { Bimanual lifting } \\
\text { rehabilitator }\end{array}$ & $\begin{array}{l}\text { treatment of } \\
\text { hemiparetic } \\
\text { patients }\end{array}$ & interactive & 1 & unknown & LUM et al. (1995) \\
\hline Cozens arm robot & $\begin{array}{l}\text { treatment of stroke } \\
\text { and MS patients }\end{array}$ & interactive & 1 & 10 & COZENS (1999) \\
\hline Arm trainer & $\begin{array}{l}\text { treatment of chronic } \\
\text { hemiparetic } \\
\text { patients }\end{array}$ & interactive & 1 & 12 & HESSE et al. (2003) \\
\hline Gentle/s system & $\begin{array}{l}\text { treatment of stroke } \\
\text { patients }\end{array}$ & interactive & $3+1$ & $>20$ & $\begin{array}{l}\text { VAN DER LINDE et al. } \\
\text { (2002); HARWIN } \\
\text { et al. }(2001) ; \text { COOTE } \\
\text { et al. }(2002 ; 2003) ; \\
\text { COOTE and STOKES } \\
\text { (2003) }\end{array}$ \\
\hline MIT-Manus & $\begin{array}{l}\text { treatment of acute } \\
\text { and chronic stroke } \\
\text { patients }\end{array}$ & interactive & $2+3$ & $>100$ & $\begin{array}{l}\text { HOGAN } \text { et al. (1995); } \\
\text { KREBS et al. }(1998) ; \\
\text { AISEN } \text { et al. }(1997) ; \\
\text { VOLPE } \text { et al. }(2000 ; \\
\text { 2002); FASOLI } \text { et al. } \\
(2003)\end{array}$ \\
\hline ARMin & $\begin{array}{l}\text { treatment of stroke } \\
\text { and SCI patients }\end{array}$ & interactive & $4+1$ & in development & $\begin{array}{l}\text { NEF and RIENER } \\
\text { (2004) }\end{array}$ \\
\hline MIME & $\begin{array}{l}\text { treatment of chronic } \\
\text { hemiparetic } \\
\text { patients }\end{array}$ & interactive & 6 & 27 & LUM et al. (2002) \\
\hline
\end{tabular}

angle. As there is no motor activity, gravity serves to assist or resist the arm movements, depending on the elevation angle of the device. A six-axis force sensor measures the interacting forces between patient and robot. Later, the device was extended by a DC servomotor to assist the movement along the linear track (Fig. 3).

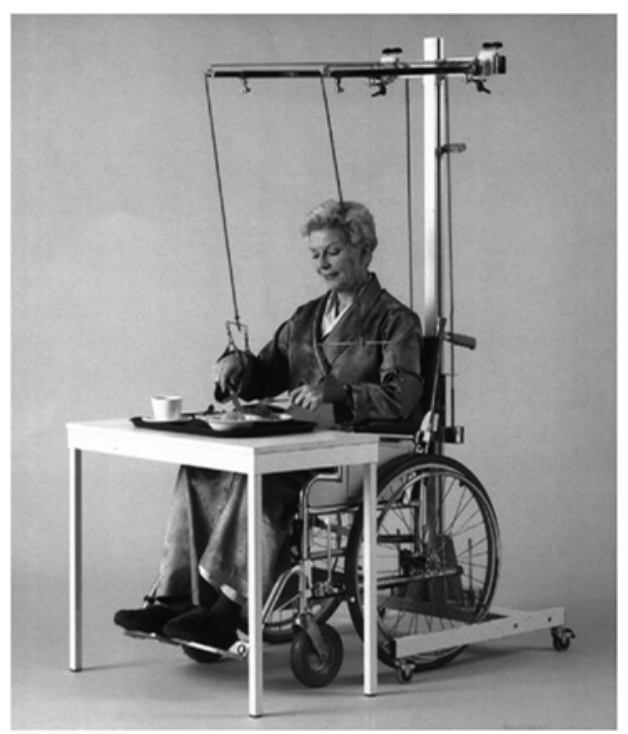

Fig. 1 Swedish Helparm (Kinsman Enterprises, Inc., with permission)

\subsection{Interactive one-DOF systems}

One of the first interactive robotic rehabilitation systems was the 'hand-object-hand rehabilitator' (LUM et al., 1993). The device consists of two vertical handles on a tabletop, each moves about an axis coincident with the subject's wrist. Both handles are connected by a stick with a force transducer that

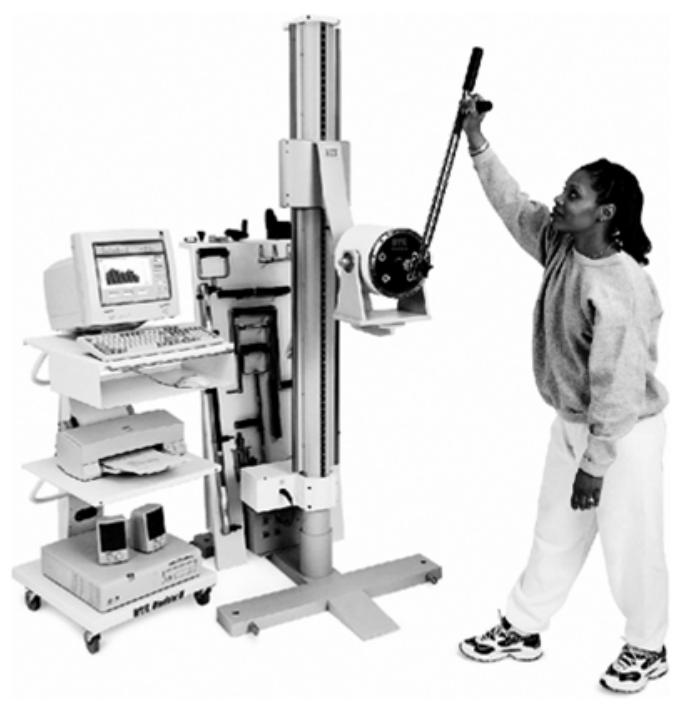

Fig. 2 BTE device Simulator II for training of ADL (photo courtesy of BTE Technologies, Inc.)

Medical \& Biological Engineering \& Computing 2005, Vol. 43 

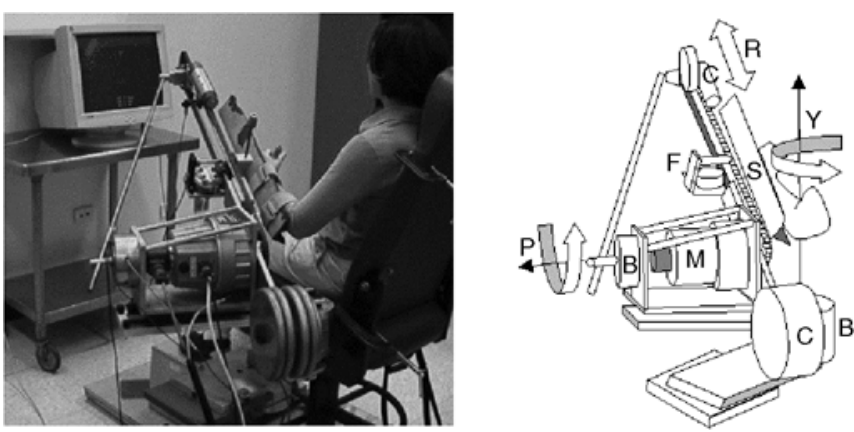

Fig. 3 Latest extended version of ARM guide (REINKENSMEYER et al. (1999a), with permission)

measures the grasp force between the outstretched fingers of the subject's hand. A potentiometer measures the position of the hands. A motor beneath one handle can produce external torque on one hand. The motivation for this configuration was that it allows bimanual tasks, with the possibility of powered assistance for one hand.

Based on the experience with the hand-object-hand rehabilitator, LUM et al. (1995) developed a similar device called the 'bimanual lifting rehabilitator'. It allows measuring and perturbing movements during the lifting of large objects, such as a cafeteria tray. The device has a handle and a force transducer for each hand attached to one rigid bar. A second bar is connected to this one, through a one-DOF bearing, and to a motor. The subject attempts to lift the link by the handles, without tilting it. A potentiometer connected to the bearing measures tilt, which is then regulated using a simple control law. Thus, if the object begins to tilt, the motor corrects, assisting the impaired hand. The bimanual lifting rehabilitator comprises one active DOF and one passive (tilt) DOF.

No clinical results have been presented with either the bimanual lifting rehabilitator or the hand-object-hand rehabilitator, so far. However, the systems served as a basis for the mirror image movement enhancer (MIME), which was used with several patients.

Another interactive device is the arm trainer developed by HESSE et al. (2003). Here, the patient has the elbow joints flexed at about $90^{\circ}$. Each hand grasps a handle and can be moved in one DOF (Fig. 4). Two handle sets are available, one with a horizontal axis for forearm pronation/supination and one with a vertical axis for wrist flexion/extension movements. The device position has to be changed depending on the selected movement. A display shows the number of cycles performed. Force and position sensors are used to enable different control modes, including position and impedance control strategies.

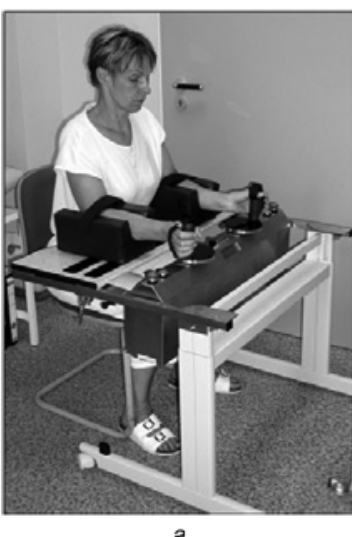

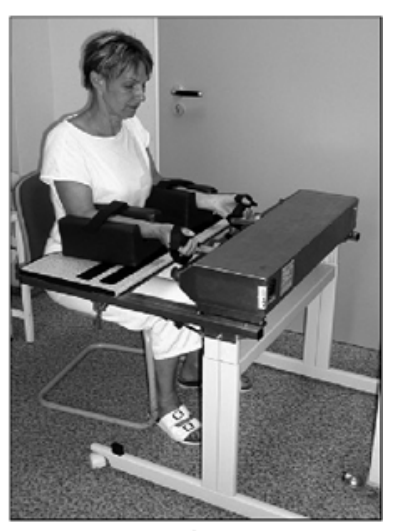

Fig. 4 Hesse arm trainer arranged in two different settings, (a) for wrist flexion and (b) for forearm pronation (HESSE et al. (2003), with permission)

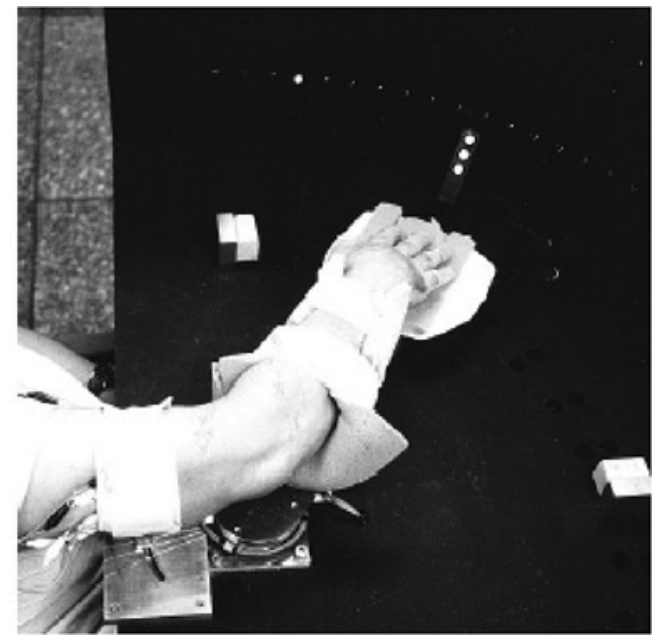

Fig. 5 Cozens arm robot (COZENS (1999), with permission)
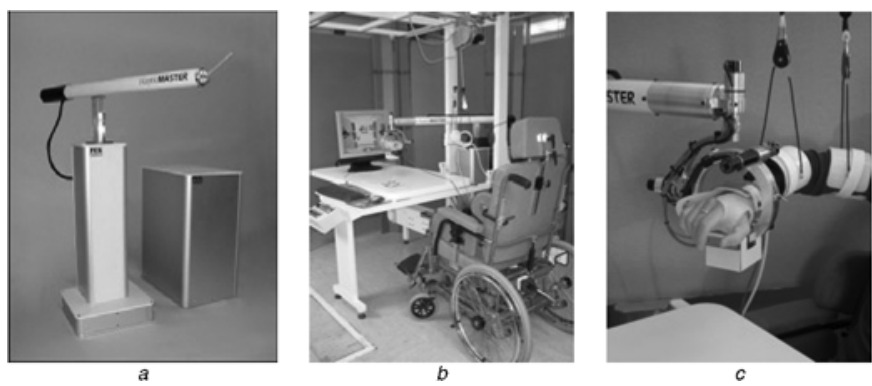

Fig. 6 (a) Haptic master (courtesy of FCS). (b), (c) As suggested by EU-funded project GENTLE/s, haptic master can be used for treatment of stroke patients (with permission)

Another one-DOF device is the arm robot from CozENS (1999). The patient's forearm is fixed to a lever that can rotate in the horizontal plane about an axis aligned with the elbow joint (Fig. 5). The patient's upper arm is constrained by straps, and therefore the device acts like an exoskeleton for the elbow joint. Interactive assistance is provided on the basis of position and acceleration signals measured by an electro-goniometer and an accelerometer. The sensor signals trigger the robot actuation as soon as a voluntary movement is being detected that is characterised by a minimum acceleration and speed. During movement, a torque controller gradually changes the amount of torque applied by the robot to avoid transforming the exercise into a pure patient-passive manipulation.

\subsection{Interactive multi-DOF systems}

The haptic master is a three-DOF robot designed as a haptic display $^{\dagger}$ (VAN DER LINDE et al., 2002). It has formed the basis of the GENTLE/s project supported by the European Union (HARWIN et al., 2001). In this project, it is suggested that the haptic master is used as a part of a rehabilitation device for the training of arm movements by attaching the wrist of the patient to the end-effector of the robot (Fig. 6). However, this set-up yields an undetermined spatial position for the elbow. Therefore two ropes of a weight-lifting system support the arm against gravity. The robot can be extended by a robotic wrist joint that provides one additional active and two passive DOFs. Force and position sensors are implemented inside the robot. Interactive support for patient movements is enabled by admittance control strategies. The system has been designed for the rehabilitation of stroke patients (COOTE et al., 2002).

${ }^{\dagger}$ Fokker Control Systems (FCS) 


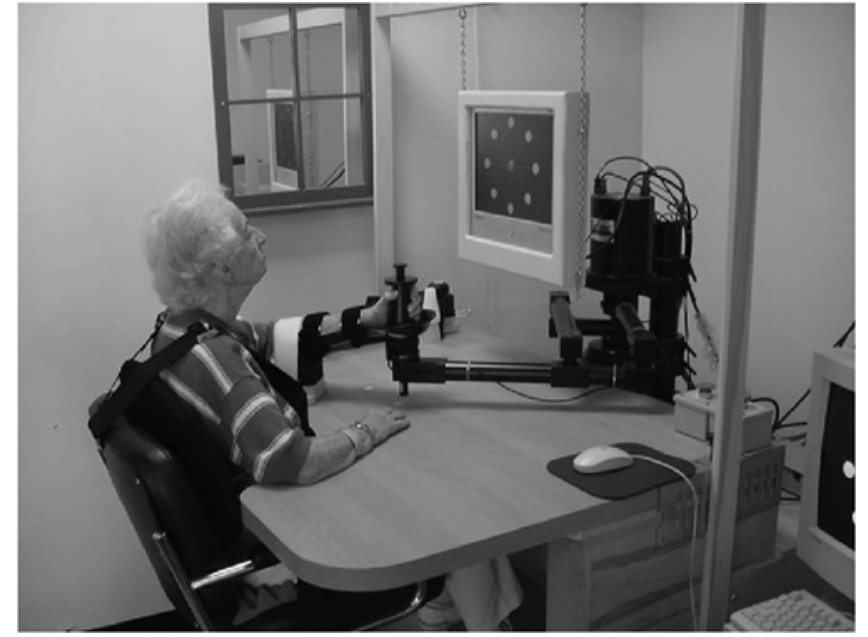

Fig. 7 Patient using MIT-Manus (HoGAN et al., 1995) (with permission from $H$. Krebs and $N$. Hogan)

One of the most advanced and commonly used arm therapy robots is the MIT-Manus (HoGAN et al., 1995; KREBS et al., 1998). It is a planar SCARA module that provides twodimensional movements of the patient's hand (Fig. 7). Forces and movements are transferred through a robot-mounted handle gripped by the patient. The MIT-Manus was designed to have a low intrinsic end-point impedance (i.e. it is backdrivable), with low inertia and friction. This design feature was emphasised primarily to facilitate control of robot impedance and to ensure that the robot's intrinsic dynamics would be minimally encumbering to the patient. Force and position sensors are used to feed the impedance controllers. A threeDOF module can be mounted on the end of the planar module, providing additional wrist motions in three active DOFs. Visual movement instructions are given by a graphic display. Clinical results for more than 100 stroke patients have been published so far (AISEN et al., 1997; VOLPE et al., 2000; 2002; FASOLI et al., 2003).

ARMin is another rehabilitation robot system currently being developed at the Swiss Federal University of Technology (ETH) and Balgrist University Hospital, both in Zurich (Fig. 8). The robot is fixed to the wall, with the patient sitting beneath. The distal part is characterised by an exoskeleton structure, with the patient's arm placed inside an orthotic shell. The current version comprises four active DOFs to allow elbow flexion/extension and spatial shoulder movements. Several multiple-axis force sensors and four position sensors enable the robot to work in different impedance control modes. A prerequisite for some of these modes is that the robot is back-drivable. The robot is designed primarily for the rehabilitation of spinal cord injured (SCI) and stroke patients.

Based on the one-DOF bimanual training devices presented above, LuM et al. (2002) developed the mirror image
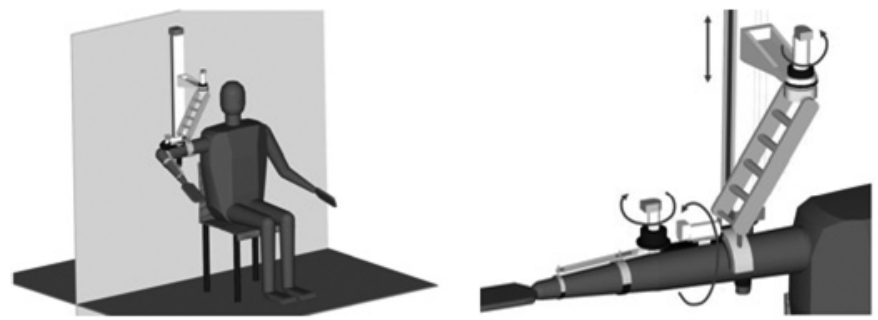

Fig. 8 Arm rehabilitation robot ARMin (NEF and RIENER, 2004)

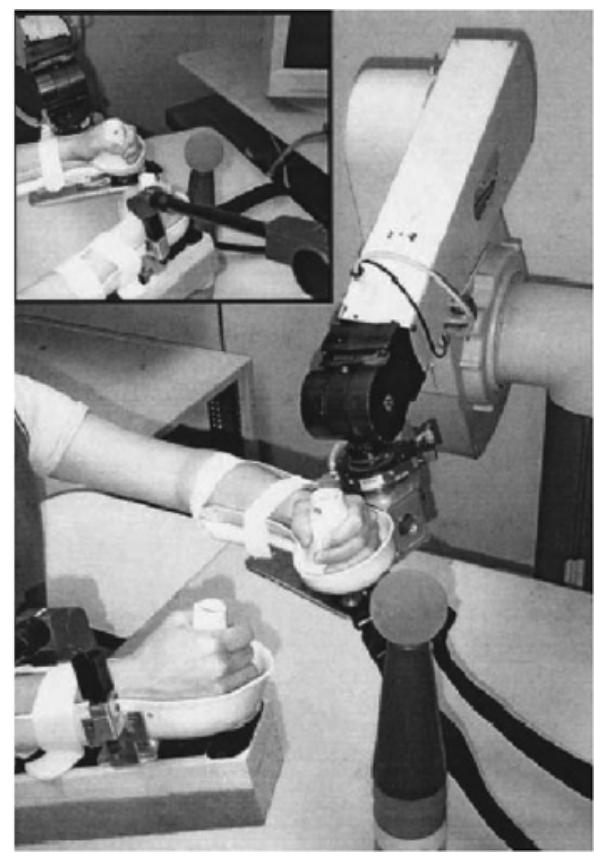

Fig. 9 Mirror image movement enhancer (LUM et al. (2002), with permission)

movement enhancer (MIME) arm therapy robot. A key element of the MIME is a six-DOF industrial robot manipulator ${ }^{*}$ that applies forces to a patient's hand that is holding a handle connected to the end-effector of the robot (Fig. 9). With this set-up, the forearm can be positioned within a large range of spatial positions and orientations. The affected arm performs a mirror movement of the movement defined by the intact arm. A six-axis force sensor and position sensors inside the robot allow the realisation of four different control modes, including position and impedance control strategies. In the 'bimanual mode', the forearms are kept in mirror symmetry by a position digitiser that measures the movement of the intact forearm and provides co-ordinates for the robot motion controller. Clinical results based on 27 subjects have been published so far.

Other groups propose the use of devices initially designed as haptic displays for virtual reality (VR) applications. One of those devices is the 'Rutgers master', which uses four pneumatic linear actuators to provide force feedback into fingers. The Rutgers master can be applied for the rehabilitation of hand and finger functions (POPESCU et al., 2000; BURDEA et al., 2000; JACK et al., 2001). Another VR device suggested for rehabilitation of hand movement is the SPIDAR system (SEAHAK et al., 1998). It consists of a rigid cubic frame and several motors with pulleys attached to every corner of the frame. Strings span from each motor-pulley unit to the subject's thumb and index finger to allow different finger movements and grips. With this system, the subject is asked to touch and move virtual objects presented by a graphical display.

\section{Clinical use}

\subsection{Clinical applicability}

Section 1.3 presented the different psychological, medical and ergonomic aspects of robot-assisted therapy and discussed the requirements from the robot. The robot approaches applied to arm therapy fulfil these requirements in different ways

\footnotetext{
${ }^{\ddagger}$ Puma 560, Stäubli Inc.
} 
(Table 2). For example, acceptance by patients is high in systems that are small. This is the case, for instance, with the Swedish Helparm, because the device is placed behind the patient so that the patient only sees the ropes connected to the wrists.

The level of patient activity is expected to be higher in those systems that are equipped with sensors and thus enable particular closed-loop (impedance) control strategies.

The feasible motions depend not only on the number of DOFs but also on the arrangement of axes, types of drive (rotational or translational) and the dimensions of the mechanical links. Eventually, the technical design and the kinds of motion possible determine whether ADL tasks can be performed with the robot or not.

A therapy device can be used for clinical evaluation, if recording of movement quantities such as angles, velocities, accelerations or forces, perhaps even EMG, is possible. Thus sensors should be integrated into the system to allow the presentation of clinical scores.

Adaptability to different body sizes is easier in end effectorbased systems, i.e. where the robot moves the arm by inducing forces only in the patient's hand (e.g. Helparm, MIME, MIT-Manus, Gentle/s system, Hesse arm robot). In contrast, exoskeletal systems are characterised by technical joint axes that are in alignment with the anatomical axes of the patient. Thus they are more difficult to adjust, because each robot link must be adapted to the corresponding patient segment (BTE devices, Cozens arm robot, ARM guide). ARMin can be considered as a mixed approach, because only the distal part is designed as an exoskeleton. The advantages of exoskeletal systems compared with the end effector-based approaches are that the arm posture is statically fully determined (i.e. known), and overstretching can be avoided by mechanical stops.

The ease of use of a robotic system can be expressed as an inverse function of its complexity. Thus robots with many DOFs and a large range of motions are more difficult and time-consuming to apply. Furthermore, the operation of exoskeletal systems requires more effort during application than end effector-based approaches, because more body segments are in contact with the device, resulting in more mechanical components that need to be adjusted and fixed. Other systems have the disadvantage that the patient has to put on special gloves or shells (e.g. Rutgers master, Cozens robot). In the Gentle/s system, owing to the statically undetermined arrangement, the arm must be supported against gravity.

\subsection{Clinical outcomes}

Only a few groups working in neurorehabilitation robotics have published relevant clinical results so far. First results were presented by AISEN et al. (1997). The group used the MIT-Manus to test whether the robotic manipulation of the impaired limb was more effective than standard rehabilitation programmes. Twenty acute hemiplegic patients, with a history of a single stroke and hemiplegia, were selected for this study. They were enrolled in a standard rehabilitation programme supplemented by either robot-aided therapy or sham robot-aided therapy. These groups were comparable in age, initial physical impairment and time between onset of the stroke and enrollment in the trial. Patients, clinical team members and the clinical evaluator were blinded to the treatment group assignments. Standardised assessment tools were applied to measure the outcomes. Impairment and

Table 2 Assessment of different robot approaches

\begin{tabular}{|c|c|c|c|c|c|c|c|}
\hline & \multicolumn{2}{|c|}{ Psychological aspects } & \multicolumn{3}{|c|}{ Medical aspects } & \multicolumn{2}{|c|}{ Ergonomic aspects } \\
\hline & $\begin{array}{c}\text { patient } \\
\text { acceptance }\end{array}$ & $\begin{array}{l}\text { level of } \\
\text { interactivity }\end{array}$ & kinds of motion & $\begin{array}{l}\text { applicability } \\
\text { of ADL tasks }\end{array}$ & $\begin{array}{c}\text { clinical scoring } \\
\text { possible }\end{array}$ & $\begin{array}{c}\text { adaptability to } \\
\text { different } \\
\text { body sizes }\end{array}$ & $\begin{array}{c}\text { convenience } \\
\text { to use }\end{array}$ \\
\hline Swedish Helparm & high & low & $\begin{array}{l}\text { 3D hand motion, } \\
\text { limited range }\end{array}$ & high & no & high & high \\
\hline BTE devices & medium & medium & $\begin{array}{l}\text { 1-DOF joint } \\
\text { motion }\end{array}$ & medium & yes & medium & medium \\
\hline ARM-guide & medium & low & $\begin{array}{c}\text { planar vertical } \\
\text { arm motion }\end{array}$ & medium & yes & medium & medium \\
\hline $\begin{array}{l}\mathrm{H}-\mathrm{O}-\mathrm{H} \\
\quad \text { rehabilitator }\end{array}$ & high & low & $\begin{array}{l}\text { rotary motion } \\
\text { around } 1 \text { axis }\end{array}$ & low & yes & high & high \\
\hline $\begin{array}{l}\text { Bimanual lifting } \\
\text { rehabilitator }\end{array}$ & high & medium & $\begin{array}{l}\text { hand motion with } \\
\text { limited range } \\
\text { and DOF }\end{array}$ & low & yes & high & high \\
\hline Cozens arm robot & medium & high & $\begin{array}{l}\text { 1-DOF elbow } \\
\text { flexion motion }\end{array}$ & low & yes & low & medium \\
\hline Hesse arm trainer & high & high & $\begin{array}{l}\text { 1-DOF hand } \\
\text { motion }\end{array}$ & medium & yes & high & high \\
\hline Gentle/s system & medium & high & $\begin{array}{l}\text { 3D arm motion, } \\
\text { limited range }\end{array}$ & medium & yes & high & medium \\
\hline MIT-Manus & medium & high & $\begin{array}{l}\text { planar horizontal } \\
\text { arm motion, } \\
\text { extendable by } \\
\text { 3-DOF wrist } \\
\text { motion }\end{array}$ & medium & yes & high & medium \\
\hline ARMin & medium & high & $\begin{array}{l}\text { 3D arm motion in } \\
4 \text { DOFs, } \\
\text { extendable } \\
\text { with } 2 \text { DOFs }\end{array}$ & high & yes & medium & medium \\
\hline MIME & low & high & $\begin{array}{l}3 \mathrm{D} \text { arm motion in } \\
6 \text { DOFs }\end{array}$ & high & yes & high & medium \\
\hline
\end{tabular}


disability declined in both groups between hospital admission and discharge. The robot-treated group showed a greater degree of improvement in all three measures of motor recovery, and the change in motor status measured in the proximal upper limb musculature was significant $(p=0.002)$. No adverse events resulted from the robot-assisted therapy.

Subsequently, VOLPE et al. (2000) performed a similar study based on the MIT-Manus. Fifty-six patients with acute stroke and hemiparesis or hemiplegia received standard post-stroke multidisciplinary rehabilitation and were randomly assigned to receive either robotic training or exposure to a robotic device without training (sham robot-aided therapy). Both the robot-treated and the control groups had comparable clinical characteristics, lesion size and pre-treatment impairment scores. By the end of the treatment, the robot-trained group demonstrated improvement in motor outcome for the trained shoulder and elbow (motor power score, $p<0.001$; motor status score, $p<0.01$ ) that did not generalise to the untrained wrist and hand. The robot-treated group also demonstrated significantly improved functional outcome (functional independence measurement-motor, $p<0.01$ ). Volpe concluded that robot-delivered quantitative and reproducible sensorimotor training enhanced the motor performance of the exercised shoulder and elbow. The robot-treated group also demonstrated improved functional outcome. When added to standard multidisciplinary rehabilitation, robotics provides novel therapeutic strategies that focus on impairment reduction and improved motor performance.

In the latest publication of the MIT-Manus group, FASOLI et al. (2003) showed that even chronic stroke patients benefit from the robotic therapy. Twenty people diagnosed with a single, unilateral stroke that had occurred within the past $1-5$ years, with persistent hemiparesis, participated in this study. Evaluations by a single blinded therapist revealed statistically significant gains between admission and discharge on the Fugl-Meyer score, motor status scale and motor power score $(p<0.05)$. Fasoli et al. concluded that robotic therapy could complement other treatment approaches by reducing motor impairment in persons with moderate to severe chronic impairments.

Another clinical study was based on the MIME device (LUM et al., 2002). The objective of this study was to compare the effects of robot-assisted movement training with conventional techniques for the rehabilitation of upper-limb motor function after stroke. Twenty-seven patients with chronic hemiparesis were randomly allocated to the two groups (robot and control group). All subjects received $241 \mathrm{~h}$ sessions over two months. Subjects in the robot group practised shoulder and elbow movements, assisted by the robot manipulator. Subjects in the control group received conventional therapy. To evaluate patient motor capabilities, the Fugl-Meyer score was evaluated by a therapist blinded to group assignments.

Compared with the control group, the robot group had greater improvements in the proximal movement portion of the Fugl-Meyer test after one month of treatment $(p<0.05)$ and also after two months of treatment $(p<0.05)$. The robot group had larger gains in strength $(p<0.02)$ and larger increases in reach extent $(p<0.01)$ after two months of treatment. At the six-month follow-up, the groups no longer differed in terms of the Fugl-Meyer test. LuM et al. (2002) concluded that, compared with conventional treatment, robotassisted movements have advantages in terms of clinical and biomechanical measures. Further research into the use of robotic manipulation for motor rehabilitation is justified.

CozENS (1999) applied his robot to ten stroke or multiple sclerosis patients aged 47-69. Each patient exhibited weakness of an upper limb, such that he or she could move the robot lever a little but was unable to complete an unassisted ten-cycle exercise with full movement range. He showed that robot assistance significantly increased the mean range of active elbow movement in every patient $(p<0.01)$.

HESSE et al. (2003) tried to determine whether use of their bilateral robotic device reduced spasticity and improved motor control in the arm of severely affected, chronic hemiparetic subjects. Twelve subjects, with a period of six months since their stroke, were investigated. They could maximally protract the affected shoulder, hold the extended arm, or slightly flex and extend the elbow. In eight subjects, a significant reduction in spasticity was noticed on the modified Ashworth scale $(p<0.0125)$ after training. However, motor control evaluated by the Rivermead motor assessment score increased only minimally in five subjects.

COOTE and STOKEs (2003) used the Haptic master to study the recovery of maximum voluntary isometric contractions in stroke patients. Their study consisted of 20 single case studies using different set-ups of the robotic system. Of the 20 patients who completed the trial, 13 showed a large and significant increase in voluntary muscle force. In another study, COOTE et al. (2003) showed, in 19 single case studies, that robot-aided therapy with the Haptic master positively affects recovery at the level of impairment and disability.

REINKENSMEYER et $a l .(1999 a ; b)$ tested the ARM guide on four hemiplegic brain-injured individuals and four unimpaired control subjects. The robot was used to quantify the arm impairment of post-stroke subjects. During guided movement, the brain-injured subjects generated distinct spatial patterns of constraint force with their impaired arms that were consistent with the standard flexion and extension 'synergies' described in the clinical literature. In addition, the impaired arms exhibited well-defined workspace deficits, as measured by the ARM guide. These results suggest that constrained force and range of motion measurements during mechanically guided movements may prove useful for precise monitoring of arm impairment and the effect of treatment techniques targeted at abnormal synergies and workspace deficits.

\subsection{Commercialisation}

Early commercial involvement with an arm therapy robot may assist in the distribution of devices and thus enable broad clinical trials, with a high number of patients and clinics involved, and ensure that a large number of patients can benefit from arm rehabilitation robots. Passive and active systems are already sold by several companies**. Among the interactive systems presented in this paper, only the MIT-

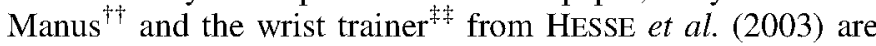
commercially available.

Reasons for the limited commercial availability of interactive systems are manifold. Most systems were developed just to show the technical feasibility. The projects are still so young so that a commercialisation could not yet be initiated. Another reason may be that the systems comprise rather complex, high-tech components (sensors, robots) that are expensive, interference-prone, accident-sensitive and maintenance-intensive. Furthermore, some groups abhor the challenging medical certification process. The use of large industrial robots in particular (such as the Puma robot) can be hazardous and requires a great effort to meet the medical device regulations.

Nevertheless, the exception proves the rule: more than 100 robotic gait training devices, such as the Lokomat* and the GaitTrainer , have been successfully sold all over the world so far.

\footnotetext{
**e.g., Kinsman Enterprises, Inc. and Baltimore Therapeutic Equipment Co.

${ }^{\dagger+}$ Interactive Motion Technologies, Inc.

${ }^{\ddagger \pm}$ Reha-Stim, Berlin

*Hocoma AG, Volketswil, Switzerland

${ }^{\dagger}$ Reha-Stim, Berlin, Germany
} 


\section{Conclusions}

This paper has presented an overview of ongoing projects, where arm rehabilitation robots are being developed and/or applied to patients. Not only technical aspects but also clinical results have been presented. Rehabilitation robots can make the therapy of upper extremity functions more efficient. The patients can train more intensively, while releasing the therapist from manual movement therapy. Thus the therapist can concentrate on other, more important aspects of the patient's treatment plan and/or take care of more patients. Furthermore, robotic systems provide accurate quantitative measurements of patient performance. Several clinical studies have shown the positive effects of robot-aided neuro-rehabilitation on the upper extremities. This may increase the acceptance of robotic systems applied in physical therapy.

It is suggested that future systems should comprise enough DOFs to allow arm movements within a reasonable range. This is required for ADL tasks and to evaluate the therapeutic outcome for a broader variety of movements. Furthermore, it is expected that patient-interactive strategies will encourage and motivate the patient, thus maximising the therapeutic outcome.

So far, most clinical investigations have been limited to stroke patients. Therefore it is recommended that robots and protocols be developed that are applicable to patients with other neurological or orthopaedic pathologies, such as incomplete spinal cord injury, multiple sclerosis, Parkinson's disease, cerebral palsy and arm pain, as well as shoulder and elbow joint lesions.

For the future, it is important that more clinical tests are performed, to prove the medical relevance of robot-aided arm therapy for different patient populations. Comparisons are necessary, not only between classical manual and automated robotic approaches, but also between several robotic devices working with different control strategies.

Acknowledgment-This study was supported in part by the NCCR for Neuroplasticity and Repair, Project 7, Switzerland.

\section{References}

Aisen, M. L., KREBS, H. I., Hogan, N., MCDowell, F., and VOLPE, B. T. (1997): 'The effect of robot-assisted therapy and rehabilitive training on motor recovery following stroke', Arch. Neurol., 54, pp. 443-446

Basmajian, J. V., Gowland, C. A., and Finlayson, M. A. (1987): 'Stroke treatment: comparison of integrated behavioural-physical therapy vs traditional physical therapy programs', Arch. Phys. Med. Rehabil., 68, pp. 267-272

Burdea, G., Popescu, V., Hentz, V., and Colbert, K. (2000): 'Virtual reality-based orthopedic telerehabilitation', IEEE Trans. Rehab. Eng., 8, pp. 430-432

COOTE, S., STOKES, E. K., AMIRABDOLLAHIAN, F., LOUREIRo, R., and HARWIN, W. (2002): 'Robot mediated therapy for the upper extremity post stroke', Irish J. Med. Sci., 170, p. 127

Coote, S., Stokes, E., Murphy, B., and Harwin, W. (2003): 'The effect of GENTLE/s robot-mediated therapy on upper extremity dysfunction post stroke'. Proc. 8th ICORR 2003, pp. 59-63

CoOTE, S., and STOKES, E. K. (2003): 'The GENTLE/s clinical trial: effect of treatment on maximal voluntary isometric contraction'. Proc. 7th Conf. AAATE Dublin, p. 78

COZENS, J. A. (1999): 'Robotic assistance of an active upper limb exercise in neurologically impaired patients', IEEE Trans. Rehab. Eng., 7, pp. 254-256

Dickstein, R., Hocherman, S., PILlar, T., and SHAHAM, R. (1986): 'Stroke rehabilitation. Three exercise therapy approaches', Phys. Ther., 66, pp. 1233-1238

Fasoli, S. E., Krebs, H. I., Stein, J. S., Frontera, W. R., and HoGAN, N. (2003): 'Effects of robotic therapy on motor impairment and recovery in chronic stroke', Arch. Phys. Med. Rehabil., 84, pp. 477-482
Feys, H. M., DE Weert, W. J., Selz, B. E., CoX Steck, G. A., SPICHIGER, R., VEREECK, L. E., PUTMAN, K. D., and VAN HOYDONCK, G. A. (1998): 'Effect of a therapeutic intervention for the hemiplegic upper limb in the acute phase after stroke: a single-blind, ramdomized, controlled multicenter trial', Stroke, 29, pp. 785-792

Harwin, W., Loureiro, R., Amirabdollahian, F., Taylor, M., Johnson, G., Stokes, E., CoOte, S., Topping, M., Collin, C. et al. (2001): 'The Gentle/s project: a new method for delivering neur-rehabilitation', in Marincek, C. et al. (Eds): Assistive technology - added value to the quality of life AAATE'01 (IOS Press, Amsterdam, 2001), pp. 36-41

Hesse, S., Schulte-Tigges, G., Konrad, M., Bardeleben, A., and WERNER, C. (2003): 'Robot-assisted arm trainer for the passive and active practice of bilateral forearm and wrist movements in hemiparetic subjects', Arch. Phys. Med. Rehabil., 84, pp. 915-920

HoGAN, N. (1985): 'Impedance control: An approach to manipulation, Parts I, II, and III', J. Dynam. Syst., Meas. Control, 107, pp. 1-23

Hogan, N., Krebs, H. I., Sharon, A., and Charnnarong, J. (1995): 'Interactive robotic therapist'. US Patent 5466213

JaCk, D., Bolan, R., MERIANS, A. S., TrEmaine, M., Burdea, G. C., ADAMOVICH, S. V., RECCE, M., and PoIZNER, H. (2001): 'Virtual reality-enhanced stroke rehabilitation', IEEE Trans. Neural Syst. Rehab. Eng., 9, pp. 308-318

JEZERnIK, S., SCHÄrer, R., COLOMbo, G., and MorarI, M. (2003): 'Adaptive robotic rehabilitation of locomotion: a clinical study in spinally injured individuals', Spinal Cord, 41, pp. 657-666

Krebs, H. I., Hogan, N., Aisen, M. L., and Volpe, B. T. (1998): 'Robot-aided neurorehabilitation', IEEE Trans. Rehab. Eng., 6, pp. $75-87$

KWAKkel, G., Wagenaar, R. C., Koelman, T. W., Lankhorst, G. J., and KoETSIER, J. C. (1997): 'Effects of intensity of rehabilitation after stroke. A research synthesis', Stroke, 28, pp. 1550-1556

KWAKKEL, G., WAGENAAR, R. C., TWISK, J. W. R., LANKHORST, G. J., and KoETSIER, J. C. (1999): 'Intensity of leg and arm training after primary middle-cerebral-artery stroke: a randomised trial', Lancet, 35, pp. 191-196

KWAKKel, G., Kollen, B. J., and WagenaAR, R. C. (2002): 'Long term effects of upper and lower limb training after stroke: a randomised trial', J. Neurol. Neurosurg. Psychiat., 72, pp. 473-479

KwEe, H., DUIMEL, J., SMIT, J., DE MOED, A. T., VAN WOERDEN, J., and KolK, L. V. D. (1988): 'The manus wheelchair-mounted manipulator: developments toward a production model'. Proc. 3rd Int. Conf. Assoc. Advancement Rehab. Technol., pp. 460-462

LANGHAMMER, B., and STANGHELLE, J. K. (2000): 'Bobarth or motor relearning programme? A comparison of two different approaches of physiotherapy in stroke rehabilitation: a randomised controlled study', Clin. Rehabil., 14, pp. 361-369

LEIFER, L. (1981): 'Rehabilitive robotics', Robot Age, pp. 4-11

LORD, J. P., and HALL, K. (1986): 'Neuromuscular re-education versus traditional programs for stroke rehabilitation', Arch. Phys. Med. Rehabil., 67, pp. 88-91

Lum, P. S., Reinkensmeyer, D. J., and LeHMan, S. L. (1993): 'Robotic assist devices for bimanual physical therapy: preliminary experiments', IEEE Trans. Rehab. Eng., 1, pp. 185-191

LuM, P. S., LEHMAN, S. L., and REINKENSMEYER, D. J. (1995): 'The bimanual lifting rehabilitator: an adaptive machine for therapy of stroke patients', IEEE Trans. Rehab. Eng., 3, pp. 166-174

Lum, P. S., Burgar, C. G., Shor, P. C., MaJMundar, M., and VAN DER LOOS, M. (2002): 'Robot-assisted movement training compared with conventional therapy techniques for the rehabilitation of upper-limb motor function after stroke', Arch. Phys. Med. Rehabil., 83, pp. 952-959

NEF, T., and RIENER, R. (2004): 'Design of the arm rehabilitation robot ARMin', Internal Report, Automatic Control Laboratory, Swiss Federal Institute of Technology (ETH), Zurich, Switzerland

Platz, T. (2003): 'Evidenzbasierte Armrehabilitation: Eine systematische Literaturübersicht', Nervenarzt, 74, pp. 841-849

Popescu, V. G., Burdea, G. C., Bouzit, M., and Hentz, V. R. (2000): 'A virtual-reality-based telerehabilitation system with force feedback', IEEE Trans. Inform. Technol. Biomed., 4, pp. $45-51$ 
REINKENSMEYER, D. J., DEWALD, J. P., and RYMER, W. Z. (1999a) 'Guidance-based quantification of arm impairment following brain injury: a pilot study', IEEE Trans. Rehab. Eng., 7, pp. $1-11$

REINKENSMEYER, D. J., SCHMIT, B. D., and RYMER, W. Z. (1999b): 'Mechatronic assessment of arm impairment after chronic brain injury', Technol. Health Care, 7, pp. 431-435

RIENER, R., and FuHR, T. (1998): 'Patient-driven control of FESsupported standing-up: a simulation study'. IEEE Trans. Rehabil. Eng., 6, pp. 113-124

SEAHAK, K., SOMSAK, W., MASAhIRO, I., YASUHARU, K., and SATO, M. (1998): 'Personal VR system for rehabilitation to hand movement'. Proc. 7th Int. Conf. on Artificial Reality and Teleexistence, pp. $102-108$

Sunderland, A., Tinson, D. J., Bradley, E. L., Fletcher, D., LANGTON, H. R., and WADE, D. T. (1992): 'Enhanced physical therapy improves recovery of arm function after stroke. A randomised controlled trial', J. Neurol. Neurosurg. Psychiat., 55, pp. $530-535$

SCHLEENBAKER, R. E., and MAINOUS, A. G. (1993): 'Electromyographical biofeedback for neuromuscular re-education in the hemiplegic stroke patient: a meta-analysis', Arch. Phys. Med. Rehabil., 74, pp. 1301-1304

Sonde, L., Gip, C., Fernaeus, S. E., Nilsson, C. G., and VITANEN, M. (1998): 'Stimulation with low frequency $(1.7 \mathrm{~Hz})$ transcutaneous electric nerve stimulation (low-tens) increases motor function of the post-stroke paretic arm', Scand. J. Rehabil. Med., 30, pp. 95-99

Taub, E., Miller, N. E., Novack, T. A., Cook, E. W., Fleming, W. C., Nepomuceno, C. S., Connell, J. S., and Crago, J. E. (1993): 'Technique to improve chronic motor deficit after stroke', Arch. Phys. Med. Rehab., 74, pp. 347-354

VAN DER LINDE, R. Q., LAMMERTSE, P., FRederiksen, E., and Ruiter, B. (2002): 'The HapticMaster, a new high-performance haptic interface'. Proc. Eurohaptics, Edinburgh, UK, pp. 1-5
VAN DER LoOs, H. F. M., Michalowski, S. J., and LeIFER, J. L. (1988): 'Development of an omnidirectional mobile vocational assistant robot'. Proc. 3rd Int. Conf. Assoc. Advancement Rehab. Technol., pp. 468-469

Volpe, B. T., Krebs, H. I., Hogan, N., Edelstein, L., Diels, C., and AISEN, M. (2000): 'A novel approach to stroke rehabilitation', Neurology, 54, pp. 1938-1944

Volpe, B. T., Ferraro, M., Krebs, H. I., and Hogan, N. (2002): 'Robotics in the rehabilitation treatment of patients with stroke', Curr. Atherosclerosis Reports, 4, pp. 270-276

WAGenaAR, R. C., MeiJer, O. G., Van Wieringen, P. C., KuiK, D. J., HAZENBERG, G. J., LINDEBOOM, J., WiCHERS, F., and RIJSWIJK, H. (1990): 'The functional recovery of stroke: a comparison between neuro-developmental treatment and the Brunnstrom method', Scand. J. Rehabil. Med., 22, pp. 1-8

ZinN, M., Roth, B., Khatib, O., and SALISBURY, J. K. (2004): 'A new actuation approach for human friendly robot design', Int. J. Robot. Res., 23, pp. 379-398

\section{Author's Biography}

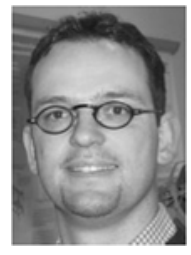

ROBERT RIENER received the Dipl.-Ing. and the Dr.-Ing. from the TU Munich in 1993 and 1997. respectively. After postdoctoral work at the Politecnico Milan, he pursued his habilitation in Biomechatronics at TU Munich. Since 2003 he has been an Assistant Professor for Rehabilitation Engineering at ETH and University Hospital Balgrist, Zurich. His current research interests involve human motion analysis, neuroprosthetics, biomechanical modelling, haptic display technologies, and rehabilitation robotics. He has authored and co-authored more than 100 journal and conference articles. He is a member of IEEE/EMBS, Automed, DHV, and a founding member and director of IFESS. 